\title{
Estudo de ovos de quatro espécies do gênero Meccus (Hemiptera, Reduviidae, Triatominae), vetores da doença de Chagas
}

\author{
Marcos Takashi Obara, José Maria Soares Barata, Nilza Nunes da Silva, \\ Walter Ceretti Júnior, Paulo Roberto Urbinatti, João Aristeu da Rosa*, \\ José Jurberg**, Cleber Galvão**/+
}

\begin{abstract}
Departamento de Epidemiologia, Faculdade de Saúde Pública, Universidade de São Paulo, São Paulo, SP, Brasil * Departamento de Ciências Biológicas, Faculdade de Ciências Farmacêuticas, Universidade Estadual Paulista, Araraquara, SP, Brasil **Laboratório Nacional e Internacional de Referência em Taxonomia de Triatomíneos, Instituto Oswaldo Cruz-Fiocruz, Av. Brasil 4365, 21045-900 Rio de Janeiro, RJ, Brasil
\end{abstract}

Morphologic, morphometrical, and histological aspects of the eggs of four species of the genera Meccus (Hemiptera, Reduviidae, Triatominae) - The objective of this work was to analyze the morphological, morphometrical, and histological characteristics of eggs of four triatomine bugs species still not studied, in order to understand phylogenetic aspects and to facilitate parameters used in taxonomy, with the purpose of a specific and generic characterization of these vectors in public health.

The eggs of each species had come from the collections of the Laboratory of Triatomine bugs and Culicid mosquitoes of the Faculdade de Saúde Pública - USP (Faculty of Public Health/USP). The morphologic studies were carried out through optic microscopy (OM) and scanning electronic microscopy (SEM). The eggs were measured with the help of the digital paquimeter Starrett ${ }^{\circledR}$ 727. In relation to histological measures, Microtome Leica RM 2145® was used. Similarities were evidenced in the exocorial architecture of the eggs when they were studied by OM and SEM. This similarity among the eggs suggests a recent speciation, probably derived from common ancestry, representing a monophyletic group. Some structures were also detected by the histological cuts.

The study has contributed to the magnification and recognition in generic and specific terms of the Triatominae subfamily. These new data will be able to subsidize a better understanding to determine roles for each vector species and to facilitate parameters to be used in taxonomy.

Key words: Triatominae - eggs - scanning electronic microscopy - morphology - morphometry - histology

O gênero Meccus foi proposto por Stål, em 1859, para uma espécie mexicana descrita em 1835 por Burmeister como Conorhinus phyllosoma. Posteriormente, esse gênero foi colocado em sinonímia com Triatoma por Pinto (1927), baseado principalmente nos artículos do rostro e inserção das antenas (Pinto 1931, Lent \& Jurberg 1982).

Mazzotti e Osório (1942) realizaram o cruzamento dessas espécies, sob condições laboratoriais. Em seus resultados, esses autores concluíram que Triatoma pallidipennis, T. picturata e T. mazzotti deveriam ser consideradas como subespécies de T. phyllosoma e propuseram a redução para o ranking de subespécies: $T$. $p$. phyllosoma; T. $p$. pallidipennis; T. $p$. picturata; T. $p$. longipennis e T. p. mazzotti. Mazzotti (1943) adicionou uma nova espécie, o T. phyllosoma usingeri. Essa seria uma forma intermediária entre $T$. $p$. picturata e $T$. p. pallidipennis.
Auxílio: Capes, Unesp, FSP/USP, CNPq, SVS/MS

+Autor de contato: clebergalvao@gmail.com

Recebido em 24 de abril de 2006

Aceito em 19 de outubro de 2006
Em 1979, Lent e Wygodzinsky destacaram a importância de análises cuidadosas para esse grupo, combinadas com intensivas coletas de exemplares. E ainda, publicaram uma lista considerando fundamentais cinco espécies, colocando em sinonímia usingeri e intermedia com longipennis.

Em 1982 Lent e Jurberg analisaram a genitália externa masculina dos machos das cinco espécies do complexo T. phyllosoma e demonstraram que várias estruturas fálicas poderiam ser utilizadas na diagnose específica: o falosoma, o suporte do falosoma, o proces-so do endosoma, a vesica, o aparelho articular e o processo mediano do pigóforo.

Em 1999, Alejandre-Aguilar et al. encontraram espécimes no ambiente silvestre, domiciliar e peridomiciliar e descreveram uma nova espécie mexicana a qual denominaram Triatoma bassolsae, afim de T. phyllosoma, usaram na diagnose entre as espécies diferenças morfológicas externas da cabeça, do rostro e da genitália.

Carcavallo et al. (2000) propuseram a revalidação do gênero Meccus com base no tamanho exagerado das espécies, em relação ao gênero Triatoma, no conexivo extremamente largo e nas diferenças encontradas na estrutura e forma dos testículos. Hypsa et al. (2002) utilizando a sistemática molecular corroboraram a proposta desta mudança taxonômica. 
Segundo Galvão et al. (2003) o presente gênero compreende hoje as seguintes espécies:

Meccus phyllosomus (Burmeister, 1835)

M. pallidipennis (Stål,1872)

M. picturatus (Usinger, 1939)

M. longipennis (Usinger, 1939)

M. mazzotti (Usinger, 1941)

M. bassolsae (Alejandre Aguilar, Nogueda Torres, Cortéz Jimenez, Jurberg, Galvão \& Carcavallo, 1999).

As espécies do gênero Meccus constituem-se vetores potenciais da doença de Chagas ao homem em várias regiões do México, devido à sua alta taxa de infecção natural por T. cruzi, produção de colônias em altas densidades e pela capacidade de domiciliação (AlejandreAguilar et al. 1993, Bautista et al. 1999, Ramsey et al. 2000, Magallón-Gastélum et al. 2001, Martínez-Ibarra et al. 2001, 2003, Espinoza-Gómez et al. 2002).

Assim, neste trabalho procuramos abordar o problema por meio de uma de nossas linhas de pesquisa que visa analisar a ultraestrutura de ovos e ninfas de Triatominae. A relevância dos estudos de ovos de Triatominae foi dada por Pinto, em 1924, quando diferenciou os ovos de Triatoma brasiliensis Neiva, 1911 de outras espécies de "barbeiros" existentes no Brasil.

Barata (1981), por meio de microscopia óptica (MO) e microscopia eletrônica de varredura (MEV) utilizou as características macroscópicas e exocoriais dos ovos como parâmetros na distinção específica de dez espécies do gênero Rhodnius. Posteriormente, em 1998, esse mesmo pesquisador ampliou esse estudo sobre a morfologia superficial dos ovos, associada às mensurações para mais nove gêneros, dentre os 15 então conhecidos gêneros de Triatominae. Rosa et al. (2000), avaliando morfométrica e morfologicamente os ovos de Triatoma rubrovaria (Blanchard, 1843) e Triatoma circummaculata (Stål, 1859) por MEV, evidenciaram a distinção entre ambas as espécies, uma vez que podem ser encontradas juntas e ocupando ecótopos semelhantes, conforme referido por Carcavallo et al. (2000).

$\mathrm{O}$ presente estudo visa à ampliação do conhecimento dos aspectos macroscópicos e exocoriais através da aná-lise das características morfológicas, morfométricas e histológicas dos ovos de quatro espécies deste gêne- ro: M. pallidipennis, M. picturatus, M. phyllosomus e M. longipennis, pretendendo explicar melhor os aspectos filogenéticos e propor a utilização de parâmetros para uso na taxonomia desse grupo.

\section{MATERIAIS E MÉTODOS}

Os ovos foram obtidos do acervo do Laboratório de Triatomíneos e Culicídeos da Faculdade de Saúde Pública da Universidade de São Paulo. Estudaram-se os ovos das seguintes procedências: $M$. pallidipennis - RCT 11 procedente do México; M. picturatus - RCT 96 procedente de Nayarit, México; M. phyllosomus - RCT 92 procedente de Nayarit, México e Meccus longipennis RCT 117 procedente de Nayarit, México.

A MO e a MEV foram utilizadas para analisar cinco amostras de cada espécie. Em MO, os desenhos, tanto do opérculo como do corpo do ovo, foram realizados em microscópio estereoscópico ZEISS ${ }^{\circledR}$-Semi SV6 e microscópio óptico binocular LEICA, ambos acoplados ao dispositivo de câmara clara. Em MEV, as amostras foram tratadas pelos métodos convencionais e fotografadas em microscópio eletrônico de varredura JEOL $\AA$ JSM T-330A.

Nos estudos morfométricos, o aparato utilizado para as mensurações foi um paquímetro digital Starrett $® 727$, sustentado por meio de um suporte de madeira previamente confeccionado.

Em cada uma dessas características buscou-se identificar diferenças entre as espécies, pelo uso da técnica de análise de variância (ANOVA), particularmente pelo teste de Tukey (Costa Neto 2002).

Nas análises histológicas, as amostras foram cortadas em micrótomo LEICA RM2145®, sendo posteriormente fotografadas com câmera NIKON FDX 35 mm.

\section{RESULTADOS}

M. pallidipennis - Em MEV, tanto o opérculo quanto o corpo do ovo apresentam na face externa estruturas exocoriais semelhantes, com áreas poligonais irregulares e de aspecto rugoso e sem ornamentações (Figs. 2A, $3 \mathrm{~A}$ e $4 \mathrm{~A})$.

M. picturatus - Apresenta o opérculo com áreas poligonais irregulares hexagonais, de aspecto ligeiramente acolchoado (Fig. 4B). O corpo do ovo apresenta
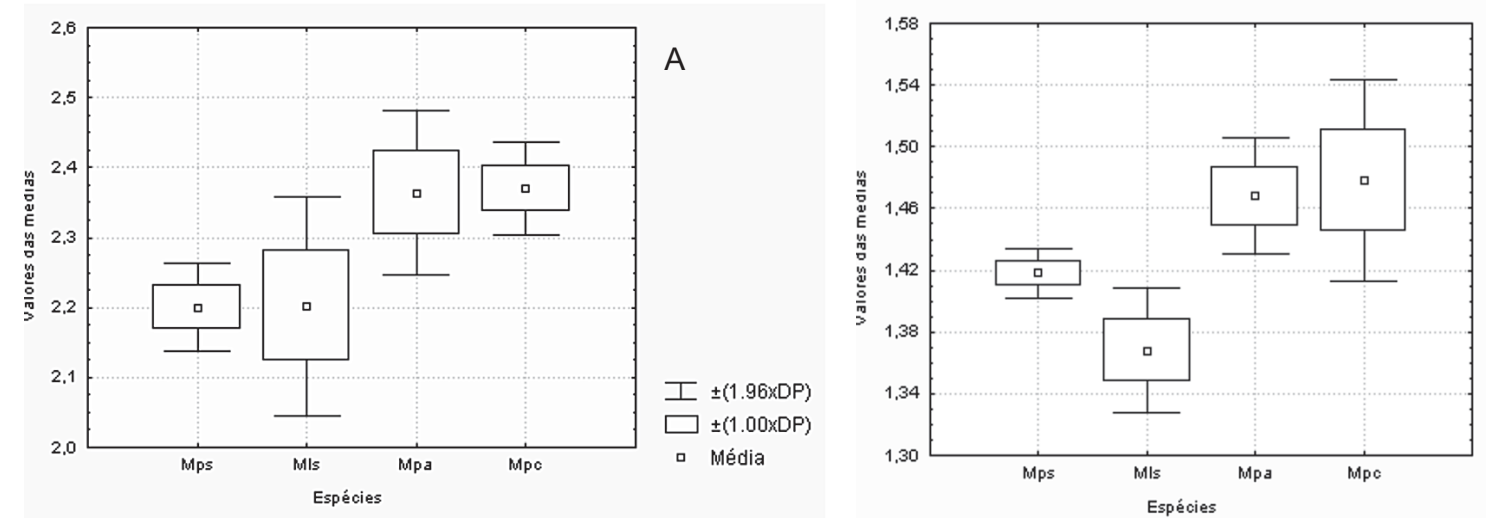

B

Fig. 1: comparações estatísticas entre espécies. A: comprimento; B: largura; Mps: M. pallidipennis; Mpc: M. picturatus; Mpa: M. phyllosomus; Mls: M. longipennis 
o exocório com áreas poligonais, a maioria hexagonais, sem ornamentações e levemente acolchoada (Figs. 2B e 3B).

M. phyllosomus - As áreas poligonais do exocório do corpo, assim como do opérculo são constituídas por estruturas hexagonais de superfície lisa e aspecto levemente acolchoado (Figs. 2C, 3C e 4C).

M. longipennis - Apresenta o exocório do corpo do ovo com presença de áreas poligonais que variam de pentagonais a heptagonais, a maioria hexagonais, ligeiramente acolchoados e de aspecto liso (Figs. 2D e 3D). O opérculo, de estrutura semelhante, é constituído por áreas poligonais hexagonais, raras pentagonais, de contorno sinuoso, sem a presença de ornamentações (Fig. 4D).

As quatro espécies apresentaram os opérculos com a forma circular convexa, simples, quase plana, simétricas e com as bordas operculares estreitas, além de bordas coriais com aspecto de uma pequena faixa estreita e com presença de aerópilas e de goteiras espermáticas em sua parte superior.

Na Tabela I são apresentados as médias e os desvios padrões das amostras analisadas. Para as médias do comprimento, é possível observar variabilidade entre 2,200 $\mathrm{mm}$ e 2,370 $\mathrm{mm}$. Na largura, as médias detectadas tiveram uma variabilidade entre 1,368 $\mathrm{mm}$ e 1,478 $\mathrm{mm}$. Considerando-se as variáveis de comprimento e largura, nas Tabelas II e III podem ser observadas as estimativas calculadas para os níveis de significância ( $\mathrm{p}<0,05 \%$ ), demonstrando diferenças significativas entre as médias das amostras estudadas. Em relação ao comprimento, os ovos de $M$. picturatus apresentaram semelhanças com os de $M$. phyllosomus, assim como os de M. longipennis para M. pallidipennis (Fig. 1).

Para a largura, observa-se que $M$. longipennis possui diferenças com $M$. picturatus e M. phyllosomus, porém, não houve diferenças significativas para as demais espécies deste gênero.

Quanto à histologia dos ovos, nas quatro espécies estudadas, é possível observar, por meio de MO a presença de quatro camadas formadoras das cascas dos ovos, que no sentido de fora para dentro, estão o exocório rijo, exocório mole, endocório mole e endocório rijo. $\mathrm{O}$ exocório rijo é a camada mais externa e formadora da estrutura externa que se deixa pouco corar pelos corantes utilizados; é imediatamente seguida pelo exocório mole que também não se deixa corar, entretanto é de espessura mais larga que o exocório rijo. $\mathrm{O}$ endocório formador da porção interna da casca é também constituído pelo endocório mole e endocório rijo; este apresenta-se como uma região larga, e deixa-se corar difusamente. Em $M$. picturatus a coloração da casca atinge tanto o endocório como parte do exocório, o que lhe dá aparência de duas faixas coradas intermediadas por faixa não corada (Fig. 5B).

Por outro lado, M. pallidipennis, M. phyllosomus e M. longipennis apresentam duas áreas homogêneas, o
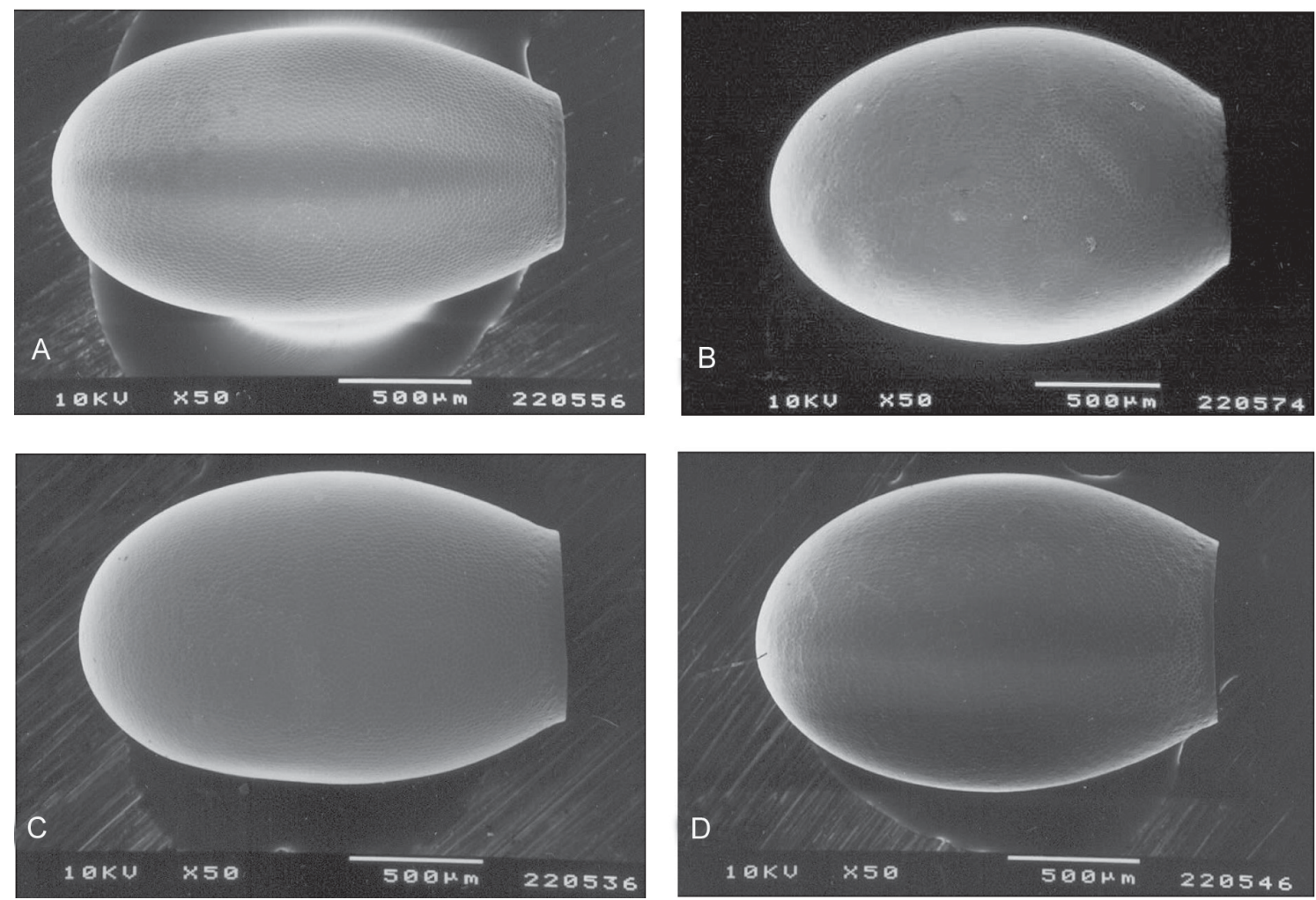

Fig. 2: aspecto geral dos ovos das espécies estudadas em microscopia eletrônica de varredura (50). A: Meccus pallidipennis; B: M. picturatus; C: M. phyllosomus; D: M. longipennis. 

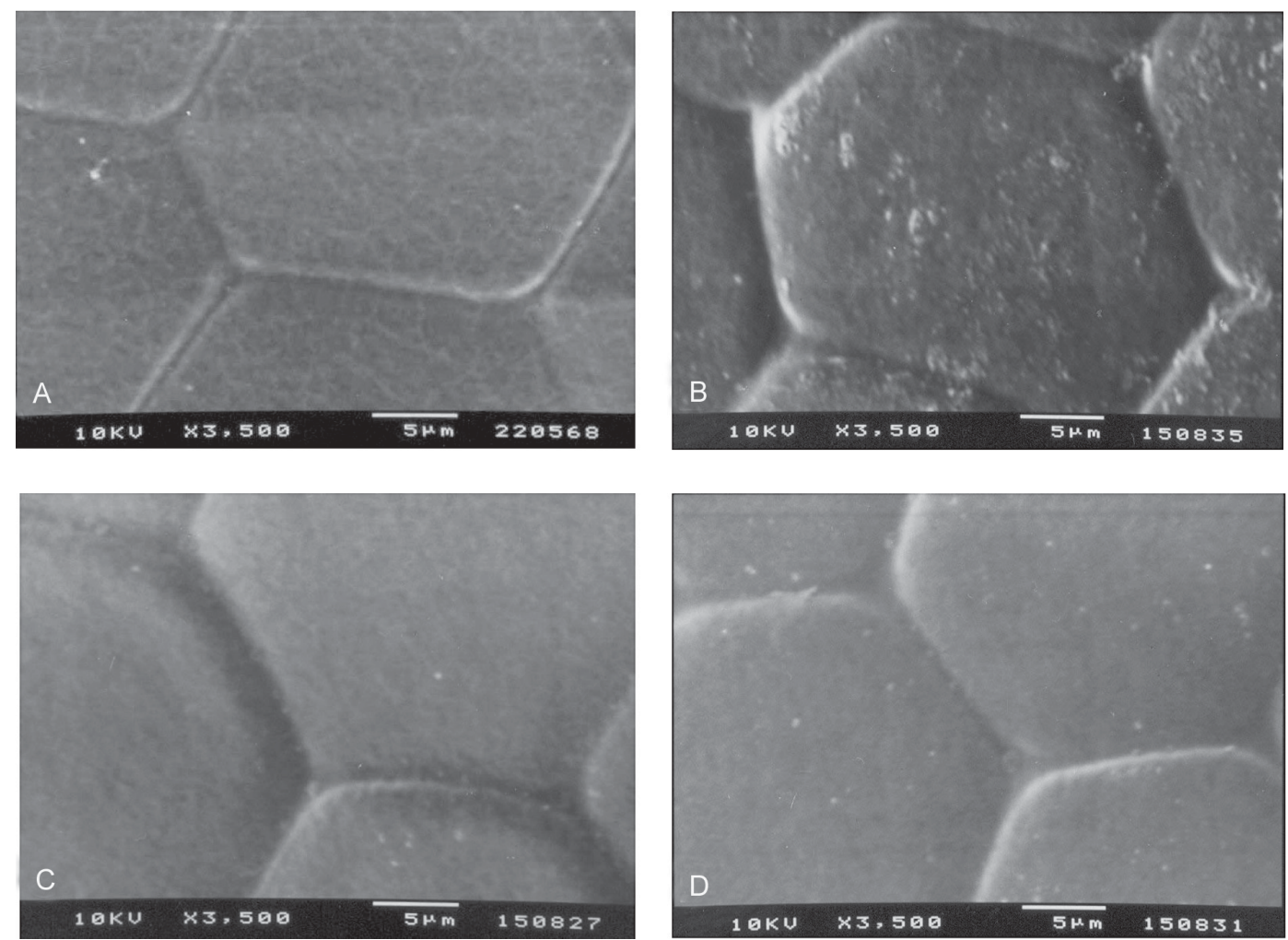

Fig. 3: detalhe da superfície exocorial das espécies estudadas em microscopia eletrônica de varredura (3500). A: Meccus pallidipennis; B: M. picturatus; C: M. phyllosomus; D: M. longipennis.
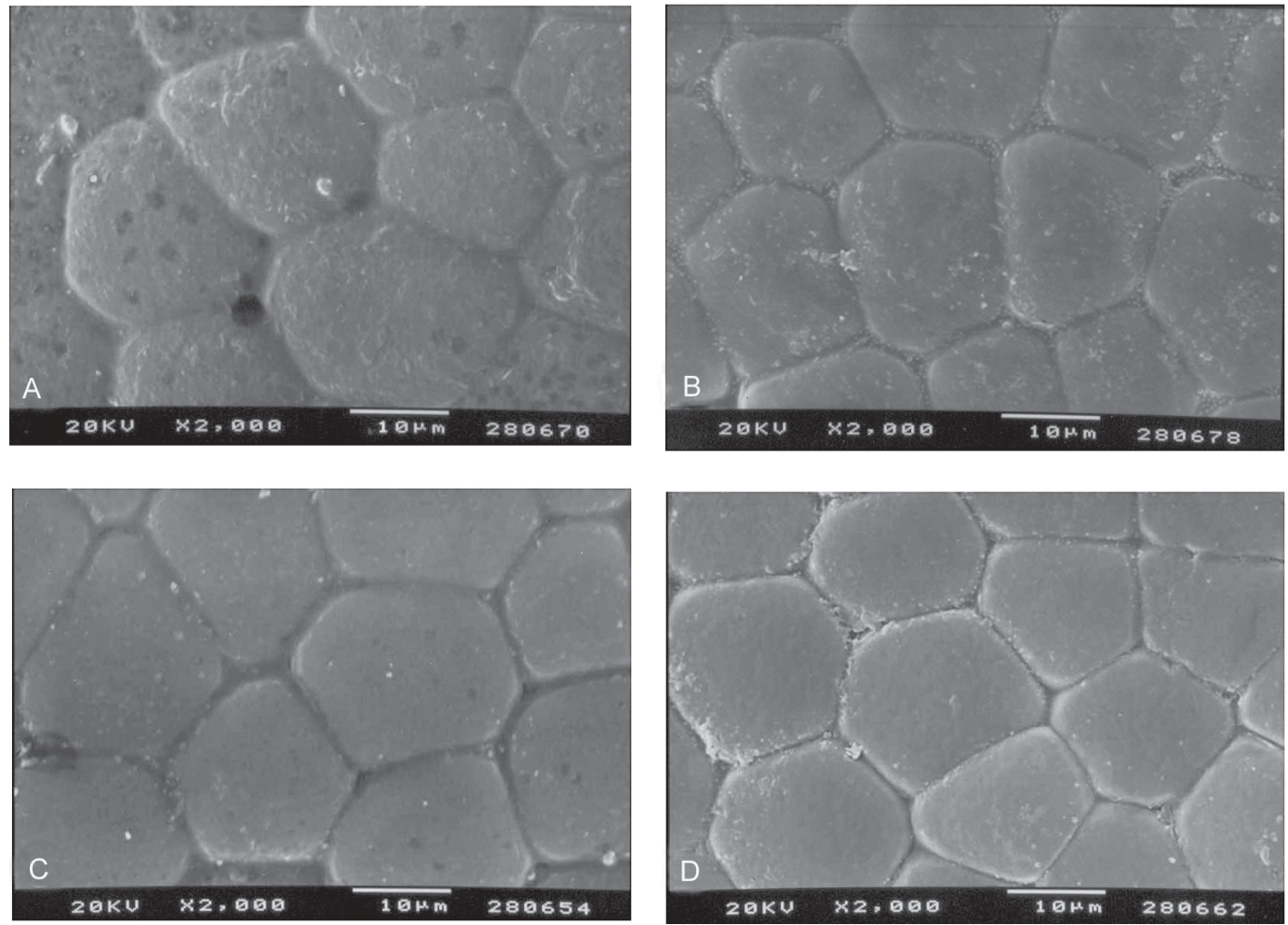

Fig. 4: detalhe da superfície exocorial do opérculo das espécies estudadas em microscopia eletrônica de varredura (2000). A: Meccus pallidipennis; B: M. picturatus; C: M. phyllosomus; D: M. longipennis. 
TABELA I

Médias $(\bar{x})$ e desvios padrões (DP) estimados do comprimento e largura $(\mathrm{mm})$

\begin{tabular}{lccccr}
\hline Espécies & \multicolumn{2}{c}{ Comprimento } & & \multicolumn{2}{c}{ Largura } \\
\cline { 2 - 3 } & \multicolumn{3}{c}{ DP } & & DP \\
\hline Meccus pallidipennis & 2,200 & 0,032 & & 1,418 & 0,008 \\
M. picturatus & 2,370 & 0,034 & & 1,478 & 0,033 \\
M. phyllosomus & 2,364 & 0,059 & & 1,468 & 0,019 \\
M. longipennis & 2,202 & 0,079 & & 1,368 & 0,020 \\
\hline
\end{tabular}

TABELA II

Resultados das diferenças entre as médias do comprimento das espécies segundo teste de Tukey

\begin{tabular}{|c|c|c|c|c|}
\hline Espécies & $\stackrel{\widehat{\Xi}}{\mathbf{\Xi}}$ & $\tilde{\Xi}$ & בิ & \\
\hline em & : & క & $\stackrel{\text { อ }}{=}$ & : \\
\hline ordem & 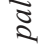 & $\cdot \widetilde{2}$ & $\frac{2}{2}$ & \\
\hline \multirow{2}{*}{ alfabética } & $\dot{z}$ & $z$ & 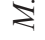 & 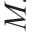 \\
\hline & $a$ & $\stackrel{2}{2}$ & $\lesssim$ & 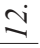 \\
\hline 9. Meccus pallidipennis & & $*$ & $*$ & \\
\hline 10. M. picturatus & $*$ & & & \\
\hline 11. M. phyllosomus & $*$ & & & $*$ \\
\hline 12. M. longipennis & & * & $*$ & \\
\hline
\end{tabular}

*diferença ao nível de significância de 5\%.

TABELA III

Resultados das diferenças entre as médias de largura das espécies segundo teste de Tukey

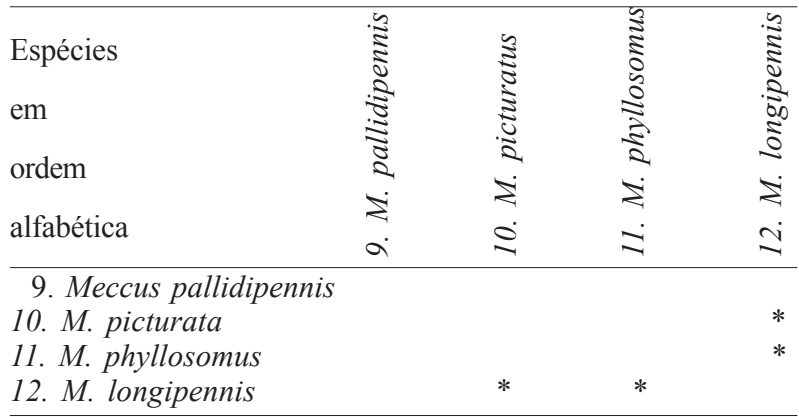

*diferença ao nível de significância de 5\%.

endocório nitidamente corado e o exocório visivelmente não corado, formando limites nítidos entre essas áreas (Figs. 5A, C, D).

Em M. picturatus, M. phyllosomus, M. longipennis e M. pallidipennis (Figs. 5A, B, C e D) nota-se que além dessas colorações, a linha formadora do exocório rijo (parte externa) apresenta-se ligeiramente ondulada, indicando a presença de células hexagonais acolchoadas.

\section{DISCUSSÃO}

Galliard (1935) foi um dos primeiros a realizar um estudo comparativo através de MO, utilizando as características ornamentais dos ovos de três gêneros: Rhodnius, Panstrongylus e Triatoma. No presente trabalho foram descritos, por meio de $\mathrm{MO}$ e MEV, as características macroscópicas e exocoriais das cascas de ovos de quatro espécies de triatomíneos; concomitantemente foram desenvolvidas observações histológicas para complementação dessa análise, assim como se realizou a comparação morfométrica do comprimento e largura desses ovos.

As quatro espécies estudadas, M. pallidipennis, $M$. picturatus, M. phyllosomus e $M$. longipennis apre-sentaram semelhanças na arquitetura exocorial dos ovos quando estudadas por $\mathrm{MO}$ e por MEV. Muito embora exibissem uma superfície externa ligeiramente rugosa em M. pallidipennis (Fig. 3A) e levemente acolchoada em M. pictutarus (Fig. 3B), comparativamente, esse grupo apresentou características exocoriais incapazes de distingui-las, principalmente entre $M$. phyllosomus e $M$. longipennis, que são nitidamente semelhantes em MEV (Figs. 3C, D).

De modo geral, embora as características individuais do exocório dessas espécies mexicanas sejam insuficientes para uma distinção específica, quando comparadas com outras espécies estudadas por Barata (1998), apresentam, de certa forma, um padrão característico para esse grupo, ou seja, arquitetura exocorial lisa, levemente separada e células hexagonais ligeiramente acolchoadas. Esse típico padrão da superfície externa dos ovos das espécies estudadas complementaria a proposta de revalidação do gênero Meccus, baseada não só na morfologia externa dos testículos, mas também no tamanho único dessas espé-cies, conforme referido por Carcavallo et al. (2000).

Essa grande afinidade desse complexo não só quanto à sua distribuição geográfica, ou seja, o território Ocidental do México, mas também do aspecto externo do inseto (abdome muito largo, tubérculos toráxicos muito salientes e ausência de fosseta tibial em ambos os sexos), da genitália masculina (Lent \& Jurberg 1982), dos padrões isoenzimáticos (Flores et al. 2001), e agora dos ovos sugerem uma especiação recente, provavelmente derivada de um ancestral comum, representando um grupo monofilético.

Quanto aos aspectos morfométricos deste grupo, verificou-se que em $M$. picturatus, tanto o comprimento $(2,370 \pm 0,034 \mathrm{~mm})$ como as larguras $(1,478 \pm 0,033$ $\mathrm{mm})$ são significantemente maiores do que as demais espécies aqui estudadas (Figs. 1A e 1B).

Barata (1981), observando os ovos de dez espécies do gênero Rhodnius detectou por meio de cortes histológicos transversais, basicamente quatro camadas. A primeira camada o exocório rijo é constituída por uma região externa larga e foi subdividida em exocório mole e exocório rijo. A segunda, camada o endocório, é constituída por uma faixa larga com cerca da metade da espessura do cório, é também formada por duas camadas: endocório mole e endocório rijo. Quanto aos aspectos histológicos das espécies aqui estudadas, as espécies do gênero Meccus apresentam características semelhantes de coloração. M. picturatus, $M$. phyllossomus e $M$. longipennis apresentam características constantes de largura entre o exocório e o endocório (Figs. 5B, C, D), sendo ligeiramente mais largo em M. pallidipennis (Fig. $5 \mathrm{~A})$. 

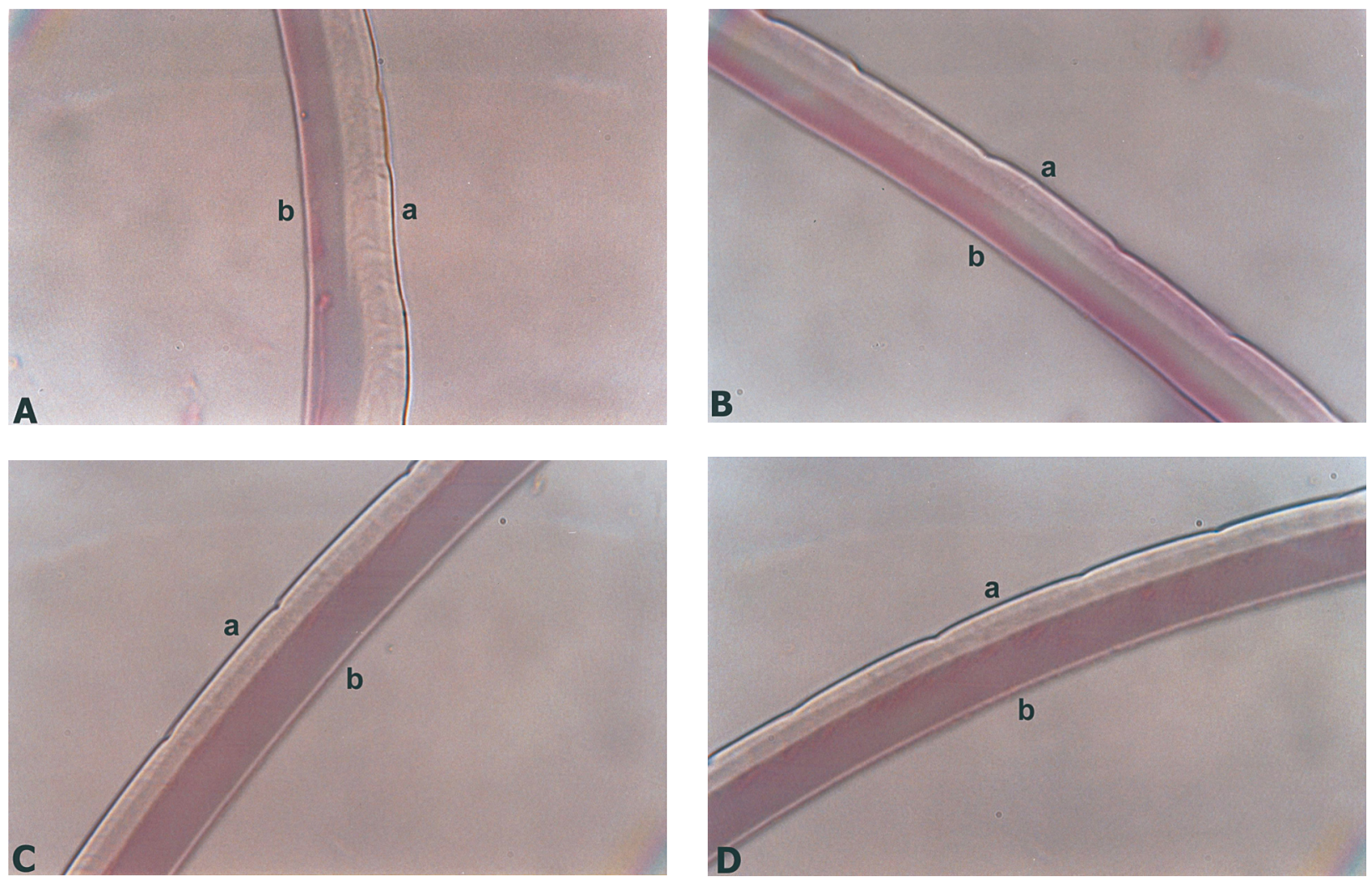

Fig. 5: fotos por meio de microscopia óptica dos cortes histológicos das cascas de ovos (100). A: Meccus pallidipennis; B: M. picturatus; C: M. phyllosomus; D: M. Longipennis; a: exocório (parte externa); b: endocório (parte interna).

Não se conseguiu definir estruturas internas no cório, entretanto o exocório em corte histológico mostrou-se com ondulações, embora pouco visíveis em fotografia (Figs. 5B, C, D), mas que certamente indicam a presença de células hexagonais acolchoadas.

Tais resultados, acrescidos aos de Carcavallo et al. (2000), talvez possam colaborar na definição sistemática do grupo reforçando a revalidação do gênero Meccus, uma vez que não foram registradas diferenças significativas entre $M$. pallidipennis, $M$. picturatus, $M$. phyllosomus e M. longipennis quanto às características morfológicas, histológicas e/ou exocoriais, o que demonstrou grande homogeneidade dentro deste grupo.

\section{AGRADECIMENTOS}

Ao Prof. Dr. Jarbas Arruda Bauer e Margareth Arroyo do Laboratório de Neurobiologia do Sistema Trigeminal do Instituto de Ciências Biológicas da USP pelo subsídio de equipamento histológico. Aos funcionários João Luis Molina Gil e João da Nóbrega do Insetário de Triatomíneos do Serviço Especial de Saúde de Araraquara-SESA, pelo envio de material entomológico para viabilização do trabalho.

\section{REFERÊNCIAS}

Alejandre-Aguilar R, Nogueda-Torres BN, Calvo-Méndez MAL, Cortéz-Jiménez M 1993. Estudio comparativo de la susceptibilidade de cinco espécies de triatominos (Insecta: Reduviidae) a la infección com Trypanosoma cruzi. Rev Lat Amer Microbiol 35: 201-206.
Alejandre-Aguilar R, Nogueda-Torres BN, Cortéz Jímenez M, JurbergJ, Galvão C, Carcavallo RU 1999. Triatoma bassolsae sp. n. do México, com uma chave para as espécies do complexo "phyllosoma" (Hemiptera, Reduviidae). Mem Inst Oswaldo Cruz 94: 353-359.

Barata JMS 1981. Aspectos morfológicos de ovos de Triatominae II. Características macroscópicas e exocoriais de dez espécies do gênero Rhodnius Stal, 1859 (Hemiptera,Reduviidae). Rev Saúde Públ 15: 490-542.

Barata JMS 1998. Macroscopic and exochorial structures of Triatominae eggs (Hemiptera, Reduviidae). In RU Carcavallo, I Galíndez-Girón, J Jurberg, H Lent (eds.), Atlas of Chagas' Disease Vectors in the Americas, Fiocruz, Rio de Janeiro, Vol. II, p. 409-448.

Bautista NL, de la Torre GSG, Arteaga IH, Schettino PMS 1999. Importance of Triatoma pallidipennis (Hemiptera: Reduvidae) as a vector of Trypanosoma cruzi (Kinetoplastida: Trypanosomatidae) in State of Morelos, México, and possibles ecotopes. J Med Entomol 36: 233-235.

Carcavallo RU, Jurberg J, Lent H, Noireau F, Galvão C 2000. Phylogeny of the Triatominae (Hemiptera: Reduviidae). Proposals for taxonomic arrangements. Entomol Vect 7: 199.

Costa Neto PLO 2002. Estatística, Edgard Blucher, São Paulo.

Espinoza-Gómez F, Maldonado-Rodríguez A, Coll-Cárdenas R, Hernández-Suárez CM, Fernández-Salas IF 2002. Presence of Triatominae (Hemiptera, Reduviidae) and risk of 
transmission of Chagas disease in Colima, Mexico. Mem Inst Oswaldo Cruz 97: 25-30.

Flores A, Magallón-Gastélum E, Bosseno MF, Ordoñez, Kasten FL, Espinosa B, Ramsey J, Brenière SF 2001. Isoenzime variability of five principal triatomine vector species of Chagas disease in Mexico. Infect Genet Evol 1: 21-28.

Galliard H 1935. Recherches sur les réduvidés hématophages Rhodnius et Triatoma. IV - Organes génitaux femelles. Ann Parasitol Hum Comp 13: 497-527.

Galvão C, Carcavallo RU, Rocha DS, Jurberg J 2003. A checklist of the current valid species of subfamily Triatominae Jeannel, 1919 (Hemiptera, Reduviidae) and their geographical distribuition, with nomenclatural and taxonomic notes. Zootaxa 202: 1-36. Disponível em www.mapress.com/ zootaxa.

Hypsa V, Tietz DF, Zrzavý J, Rego ROM, Galvão C, Jurberg J 2002. Phylogeny and biogeography of Triatominae (Hemiptera: Reduviidae): molecular evidence of a New World origin of the Asiatic clade. Mol Phyl Evol 23: 447-457.

Lent H, Jurberg J 1982. A genitália externa dos machos no complexo Triatoma phyllosoma (Burm.) espécies mexicanas transmissoras da doença de Chagas (Hemiptera, Reduviidae). Mem Inst Oswaldo Cruz 77: 299-317.

Lent H, Wygodzinsky P 1979. Revision on the Triatominae (Hemiptera,Reduviidae) and their significance as vectors of Chagas'disease. Bull Am Mus Nat Hist 163: 123-520.

Magallón-Gastélum E, Lozano-Kasten, Flores-Pérez, Bosseno MF, Brenière SF 2001. Sylvatic Triatominae of the Phyllosoma Complex (Hemiptera, Reduviidae) around the community of Carrillo Puerto, Nayarit, Mexico. Med Vet Entomol 38: 638640.

Martínez-Ibarra JA, Barcenas-Ortega NM, Nogueda-Torres B,
Alejandre-Aguilar R, Rodríguez ML, Magallón-Gastélum E, López-Martínez V, Romero-Nápoles J 2001. Role of two Triatoma (Hemiptera: Reduviidae: Triatominae) species in the transmission of Trypanosoma cruzi (Kinetoplastida: Trypanosomatidae) to man in the west coast of Mexico. Mem Inst Oswaldo Cruz 96: 141-144.

Matínez-Ibarra JA, Novelo-López M, Hernández-Robles MR, Grant-Guillén Y 2003. Influence of the blood meal source on the biology of Meccus picturatus Usinger, 1939 (Hemiptera: Reduviidae: Triatominae) under laboratory conditions. Mem Inst Oswaldo Cruz 98: 227-232.

Mazzotti L 1943. Triatoam phyllosoma usingeri, a new subspecies of Triatoma from Mexico. Pan Pac Entomol 19: 80-85.

Mazzotti L, Osório MT 1942. Cruzamientos experimentales entre varias espécies de triatomas. Rev Mex Med 412: 215 222.

Pinto C 1924. Biologia do Triatoma brasiliensis Neivai. Sciencia Médica 2: 541-543.

Pinto C 1927. Classification de genres d'hemiptères de la famille Triatomidae (Reduvidioidea). Bol Biol 8: 103-114.

Pinto C 1931. Valor do rostro e das antenas na caracterização dos gêneros de triatomideos. Hemiptera. Reduvidioidea. Bol Biol 19: 45-136.

Ramsey JM, Ordoñez R, Cruz-Celis A, Alvear AL, Chavez V, Lopez R, Pinto JR, Gama F, Carrillo S 2000. Distribution of domestic Triatominae and stratification of Chagas disease transmission in Oaxaca, Mexico. Med Vet Entomol 14: 1930 .

Rosa JA da, Barata JMS, Santos JLF, Cilense M 2000. Morfologia de ovos de Triatoma circummaculata e Triatoma rubrovaria (Hemiptera, Reduviidae). Rev Saúde Públ 34: 538-542. 